\title{
Oral health in the Japan self-defense forces - a representative survey
}

\author{
Yuka Kudo', Mike T John², Yoko Saito ${ }^{1}$, Shachi Sur ${ }^{1}$, Chisako Furuyama ${ }^{1}$, Hiroaki Tsukasaki ${ }^{1}$ and Kazuyoshi Baba ${ }^{1 *}$
}

\begin{abstract}
Background: The oral health of military populations is usually not very well characterized compared to civilian populations. The aim of this study was to investigate two physical oral health characteristics and one perceived oral health measure and their correlation in the Japan self-defense forces (JSDF).
\end{abstract}

Methods: Number of missing teeth, denture status, and OHRQOL as evaluated by the Japanese 14-item version of the Oral Health Impact Profile (OHIP-J14) as well as the correlation between these oral health measures was

investigated in 911 personnel in the JSDF.

Results: Subjects did not have a substantial number of missing teeth and only $4 \%$ used removable dentures. The mean OHIP-J14 score was $4.6 \pm 6.7$ units. The magnitude of the correlation between the number of missing teeth with OHIP-J14 scores was small $(r=0.22, p<0.001)$. Mean OHIP-J14 scores differed between subjects with and without dentures (8.6 and 4.4, $p<0.001$ ).

Conclusions: Compared to Japanese civilian populations, personnel of the JSDF demonstrated good oral health. Two physical oral health characteristics were associated with perceived oral health.

\section{Background}

In many countries oral health of the general population and of various patient populations is well characterized. However, in military populations it is often not characterized even if oral health is considered an important part of general health and therefore influences the health status of military personnel and their ability to perform their duties. Furthermore, their good oral health would decrease the number of urgent dental interventions and absences from training and the battlefield that would in turn, improve the security of the whole formation [1].

Oral health has two dimensions. First, there is the physical oral health status in terms of number of teeth, periodontal status, mouth opening etc. Second, how the individual perceives his or her oral health is equally important. Both dimensions are needed to characterize oral health comprehensively.

Key characteristics of physical oral health are the number of teeth and denture status and such findings are standard components of oral health surveys for nonmilitary populations [2-5]. For example, one study that

\footnotetext{
* Correspondence: kazuyoshi@dent.showa-u.ac.jp

${ }^{1}$ Department of Prosthodontics, Showa University, Japan

Full list of author information is available at the end of the article
}

investigated a general population, age ranging 20 to 59 years old, reported that $49.0 \%$ of the subjects had intact dental arch with no missing teeth, while $39.0 \%$ had 1 to 4 and $12.1 \%$ had 5 or more missing teeth and that the majority of these subjects with missing teeth had fixed partial dentures and only a small number of subjects had removable partial dentures (7\%) [6].

The most comprehensive concept describing perceived oral health is oral health related quality of life (OHRQoL), which has been recognized more and more frequently as an important component of health [7]. Therefore, collecting OHRQoL information in oral health surveys is increasingly performed to provide complementary information in addition to physical oral health indicators [8-10]. One of the instruments frequently used to measure OHRQoL is the Oral Health Impact Profile (OHIP) questionnaire [7], which asks about the impact of oral conditions on everyday wellbeing. The OHIP questionnaires have been translated into various languages in both full and abbreviated versions, ranging from 49 to 5 items [7,11-14]. A Japanese long version (OHIP-J54) [15] and a short version (OHIP-J14) [16] have recently been developed.
C Biomed Central

() 2011 Kudo et al; licensee BioMed Central Ltd. This is an Open Access article distributed under the terms of the Creative Commons Attribution License (http://creativecommons.org/licenses/by/2.0), which permits unrestricted use, distribution, and reproduction in any medium, provided the original work is properly cited. 
For military personnel's oral health, some reports are available in the literature $[1,17,18]$; however no data has been published so far for the Japan self-defense forces.

The aim of this study was to investigate two physical oral health characteristics (number of missing teeth and denture status) and one perceived oral health measure (oral health related quality of life, OHRQoL) and their correlation in the Japan self-defense forces (JSDF).

\section{Methods}

Subjects were consecutively sampled from a Camp of the JSDF during the annual medical examination in 2008 (mean aged $35.7 \pm 10.1$, range $15-59$ years). Nine hundred and eleven individuals agreed to participate in this study and gave informed consent.

To assess physical oral health, tooth status of the participants was examined by a single dentist and recorded as present or absent. For analysis, missing teeth were categorized $(0,1$, or $2+)$, while the fixed prostheses were regarded as the teeth were present. Denture status was categorized as fixed or removable prostheses.

To assess perceived oral health, oral health-related quality of life was evaluated using OHIP-J14 [16]. For each of the 14 OHIP questions, subjects were asked how frequently they had experienced impact in the preceding 12 months and coded as $4=$ very often, $3=$ fairly often, $2=$ occasionally, $1=$ hardly ever and $0=$ never. The responses were summed up into a score ranging from 0 to 56. A score of 0 indicated no perceived oral health problem and 56 indicated maximum impairment.

To measure how physical and perceived oral health are related, a Pearson correlation coefficient assessed the correlation between number of missing teeth and OHIPJ14 scores and a point-biserial correlation coefficient assessed the correlation between denture status and OHIP-J14 scores. Subgroup correlation analyses were performed in 4 age groups and gender. A t-test analyzed the difference in the mean OHIP-J14 scores between subjects with and without removable partial dentures.

The study protocol was conducted in compliance with the Helsinki Declaration and was approved by the Ethics Committees of Showa University (\#2007-29).

\section{Results}

\section{Subject characteristics}

Subjects had a mean age of 35.7 years (SD: 10.1, min: 15, max: 59 years) and were predominantly male (Table 1 ).

\section{Number of teeth and denture status}

The subjects did not have many missing teeth [mean: 0.85 , prevalence of subjects with at least one (and not with fixed partial dentures replaced) missing tooth: $28 \%]$. The majority of subjects $(\mathrm{N}=678,74 \%)$ had neither fixed nor removable dentures. Among the
Table 1 Demographics

\begin{tabular}{lcc}
\hline Characteristics & N & $\%$ \\
\hline Age & & \\
$15-24$ yrs & 161 & 17.7 \\
$25-34$ yrs & 284 & 31.2 \\
$35-44$ yrs & 258 & 28.4 \\
$45-59$ yrs & 207 & 22.8 \\
Females & 60 & 6.6 \\
\hline
\end{tabular}

remaining 233 subjects, only 38 subjects had removable partial dentures and one subject had complete dentures, totaling $4 \%$ of the whole sample (Table 2). The data form the subject with complete dentures was integrated in the group of removable dentures for the analyses. Among age and gender characteristics, age was the strongest factor differentiating tooth loss and denture status.

\section{Oral health-related quality of life}

The subjects did not suffer from major OHRQoL impairment. Only 0.2 to $1.9 \%$ reported frequent negative impacts (response categories fairly often or very often) with mean scores for those items ranging from 0.24 to 0.41 (Table 3). The most commonly reported impact was within the dimension of 'physical discomfort', 1.9\% reported being self-conscious fairly often or very often. The mean OHIP score for all subjects was $4.6+/-6.7$ $(95 \%$ confidence interval $=4.1-5.0$, Table 4$)$.

\section{Correlation between physical characteristics of oral health and perceived oral health}

The magnitude of the correlations between physical oral health characteristics and perceived oral health as measured by OHRQoL was trivial or small. The relationship

Table 2 Number of missing teeth and frequency of denture types for all subjects and stratified by age and gender

\begin{tabular}{|c|c|c|c|c|c|c|}
\hline \multirow{2}{*}{\multicolumn{2}{|c|}{ characteristic }} & \multicolumn{3}{|c|}{ Missing teeth } & \multicolumn{2}{|c|}{ Denture Status } \\
\hline & & 0 & 1 & $2+$ & $\begin{array}{c}\text { No } \\
\text { Denture }\end{array}$ & $\begin{array}{c}\text { Removable } \\
\text { Denture }\end{array}$ \\
\hline & $\mathrm{N}$ & & & & & \\
\hline $\begin{array}{l}\text { all } \\
\text { subjects }\end{array}$ & 911 & 72.5 & 12.1 & 15.5 & 95.7 & 4.3 \\
\hline
\end{tabular}

\begin{tabular}{|c|c|c|c|c|c|c|}
\hline $15-24$ yrs & 161 & 88.8 & 4.4 & 6.8 & 100.0 & 0.0 \\
\hline $25-34$ yrs & 284 & 83.1 & 9.9 & 7.0 & 99.6 & 0.4 \\
\hline $35-44$ yrs & 258 & 62.8 & 16.7 & 20.5 & 95.7 & 4.3 \\
\hline $45-59$ yrs & 207 & 57.5 & 15.0 & 27.5 & 87.0 & 13.0 \\
\hline male & 850 & 72.0 & 12.6 & 15.4 & 95.0 & 5.0 \\
\hline female & 60 & 80.0 & 3.3 & 16.7 & 95.8 & 4.2 \\
\hline
\end{tabular}


Table 3 Distribution of responses (\%)

\begin{tabular}{|c|c|c|c|c|}
\hline $\begin{array}{l}\text { Dimension and description of item 'Because of problems with your teeth, } \\
\text { mouth or dentures, during the last } 1 \text { months,...' }\end{array}$ & $\begin{array}{c}\text { Never(0)/ } \\
\text { hardly ever(1) }\end{array}$ & $\begin{array}{l}\text { Occasionally } \\
\text { (2) }\end{array}$ & $\begin{array}{l}\text { Fairly often(3)/ } \\
\text { very often(4) }\end{array}$ & Mean \\
\hline \multicolumn{5}{|l|}{ Functional limitation } \\
\hline Have you had trouble pronouncing any words? & 94.4 & 4.6 & 1.0 & 0.3 \\
\hline Have you felt that your sense of taste has worsened? & 97.3 & 2.3 & 0.4 & 0.2 \\
\hline \multicolumn{5}{|l|}{ Physical pain } \\
\hline Have you had painful aching in your mouth? & 92.6 & 6.2 & 1.2 & 0.4 \\
\hline Have you found it uncomfortable to eat any foods? & 92.7 & 5.9 & 1.4 & 0.4 \\
\hline \multicolumn{5}{|l|}{ Psychological discomfort } \\
\hline Have you been self-conscious? & 90.5 & 7.6 & 1.9 & 0.4 \\
\hline Have you felt tense? & 93.5 & 5.7 & 0.8 & 0.4 \\
\hline \multicolumn{5}{|l|}{ Physical disability } \\
\hline Has your diet been unsatisfactory? & 95.5 & 4 & 0.5 & 0.3 \\
\hline Have you had to interrupt meals? & 97.5 & 2.3 & 0.2 & 0.2 \\
\hline \multicolumn{5}{|l|}{ Psychological disability } \\
\hline Have you found it difficult to relax? & 93.4 & 5.1 & 1.5 & 0.3 \\
\hline Have you been a bit embarrassed? & 95.0 & 4.1 & 0.9 & 0.4 \\
\hline \multicolumn{5}{|l|}{ Social disability } \\
\hline Have you been a bit irritable with other people? & 93.7 & 5.1 & 1.2 & 0.4 \\
\hline Have you had difficulty doing your usual jobs? & 96.7 & 2.8 & 0.5 & 0.3 \\
\hline \multicolumn{5}{|l|}{ Handicap } \\
\hline Have you felt that life in general was less satisfying? & 94.7 & 4.0 & 1.3 & 0.4 \\
\hline Have you been totally unable to function? & 97.0 & 2.3 & 0.7 & 0.3 \\
\hline
\end{tabular}

between number of missing teeth and OHIP-J14 scores was nearly linear fitting a flexible curve to the data (locally weighted scatter plot smoothing, lowess) in Figure 1. Describing the linear relationship with the Pearson correlation coefficient showed a small but statistically significant correlation of $0.22(\mathrm{P}<0.001)$. Even mean OHIP-J14 scores differed by denture status subgroups $(\mathrm{p}<0.001$, t-test, Figure 1$)$, the point-biserial correlation coefficients between denture status (yes/no) and OHIP-J14 scores was small with $0.17(\mathrm{P}<0.001)$.

Table 4 OHIP-J14 summary score for all subjects and stratified by age and gender

\begin{tabular}{|c|c|c|c|}
\hline Characteristic & & $\begin{array}{l}\text { OHIP mean } \\
(S D)\end{array}$ & $\begin{array}{c}\text { 95\% confidence interval for the } \\
\text { mean }\end{array}$ \\
\hline all subjects & & $4.6(6.7)$ & $4.1-5.0$ \\
\hline \multicolumn{4}{|l|}{ age groups } \\
\hline $15-24$ yrs & 161 & $4.1(6.9)$ & $3.0-5.1$ \\
\hline $25-34$ yrs & 285 & $3.0(5.3)$ & $2.4-3.6$ \\
\hline $35-44$ yrs & 258 & $5.3(6.7)$ & $4.4-6.1$ \\
\hline 45-59 yrs & 207 & $6.1(7.6)$ & $5.1-7.1$ \\
\hline Males & 851 & $4.5(6.7)$ & $4.1-5.0$ \\
\hline Females & 60 & $4.9(6.8)$ & $3.1-6.6$ \\
\hline
\end{tabular}

When investigated in the levels of the two sociodemographic variables (Table 5), all subgroup correlations for number of missing teeth and OHIP-J14 were $r \leq 0.30$ and all subgroup correlations for denture status and OHIP-J14 were $r \leq 0.41$ - a magnitude of the

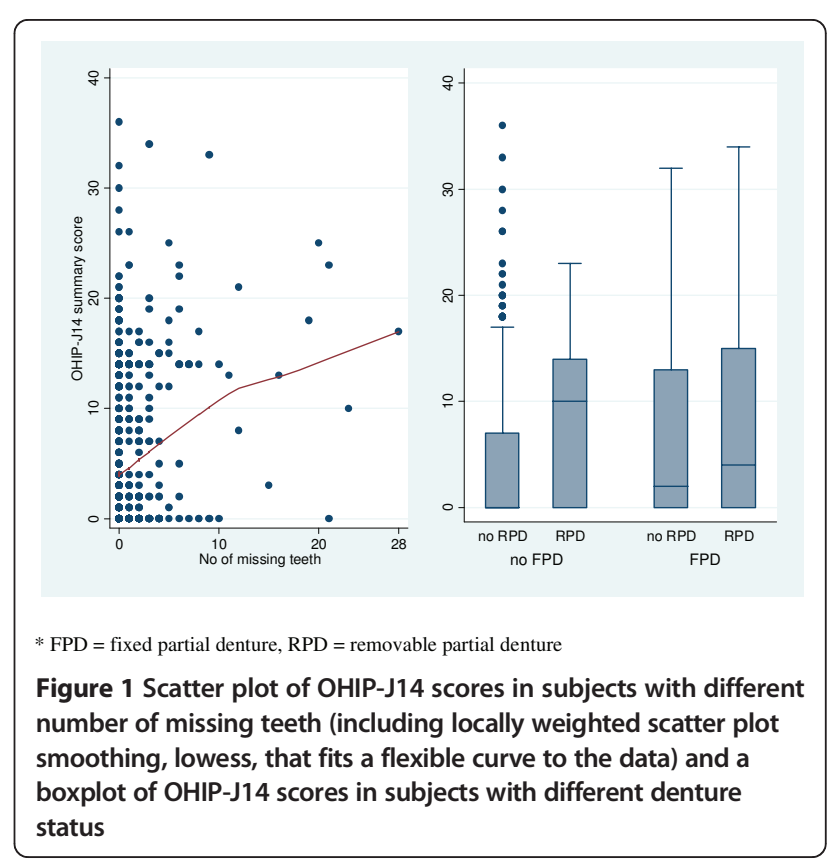


Table 5 Pearson correlation coefficient between OHIP-J14 summary score and number of missing teeth and pointbiserial correlation coefficient between OHIP-J14 summary score and denture status (with RPD/without RPD)

\begin{tabular}{|c|c|c|c|c|c|}
\hline Characteristic & & Pearson correlation coefficient & $P$ value & Point-biserial correlation coefficient & $P$ value \\
\hline \multicolumn{6}{|l|}{ age groups } \\
\hline $15-24$ yrs & 161 & 0.06 & 0.49 & 0.05 & 0.55 \\
\hline $25-34$ yrs & 285 & 0.05 & 0.35 & 0.10 & 0.10 \\
\hline $35-44$ yrs & 258 & 0.25 & 0.01 & 0.09 & 0.16 \\
\hline 45-59 yrs & 207 & 0.25 & 0.01 & 0.15 & 0.03 \\
\hline Males & 851 & 0.22 & 0.01 & 0.17 & 0.00 \\
\hline Females & 60 & 0.27 & 0.04 & 0.24 & 0.06 \\
\hline
\end{tabular}

RPD $=$ Removable Partial Denture.

correlations that would be considered "medium" according to Cohen [19].

\section{Discussion}

We found good oral health, both physical, i.e., when assessed by a dentist, and perceived by the individual, in the Japan self-defense forces (JSDF). When searching the literature to compare our results, we found only a limited number of oral health reports in military populations $[1,17,18]$. We did not find a study assessing OHRQoL in military populations even though this concept has been increasingly recognized as an important component of health.

Our results suggest that the magnitude of correlations between physical characteristics of oral health and perceived oral health is small in this military population. However, because both "dimensions" of oral health affect the military personnel's readiness, assessment of physical and perceived health is necessary and we recommend including a measure of perceived oral health when military personnel oral health is examined. The only limited information available is on the missing tooth number of the military populations in other countries. They reported that the average number of missing teeth for the Croatian army [1] was 2.3 for 650 recruits and 5.1 for 262 professionals (all male, averaged age = 32.7 years, age range 18 to 54 years) and that for Danish military [18] was 0.02 to 0.5 (all male, average age $=$ 25.2, age range 19 - 49 years). Our results are lower than the Croatian results and higher than the Danish results. However, the Danish subjects were younger and we found an age influence on the missing number of teeth which is also supported from studies done in civilian populations.

When compared with non-military populations, it was reported that $76.7 \%$ of the population did not have any missing tooth in New Zealand [20]. Some studies reported lower numbers such as $62.3 \%$ in India [21] and $53.5 \%$ in Israel [17]. Although direct comparison with our study findings is not possible due to the difference in age and gender distribution and tooth counting system, these data provide a general framework of how prevalent tooth loss is in the general population. Japanese population-based studies reported that $49 \%$ of subjects had intact dental arch with no missing teeth and the average number of missing teeth was 1.3 (age range 15 - 59 years) $[6,22]$, which is higher than the result of this study.

Regarding denture status, the other key characteristic of physical oral health that we investigated, the Danish Military study $(\mathrm{n}=223$, all men, average age $=25.2$ years, age range 19 - 49 years) reported no subject used removable dentures, which is lower than the current study result (4\%). This might be due to the difference in the age of the studied populations. The denture status investigations in population based samples in Germany [12], Finland [23] and Malaysia [13] reported that $19 \%$ used removable partial dentures and 5\% used complete dentures in Germany (average age $=43.3$ years), that $18 \%$ used removable partial dentures and 12\% used complete dentures in Finland (age $>30$ years), and that $16.7 \%$ used removable dentures in Malaysia (age data not available). Again, a direct comparison is difficult to make because of methodological study differences. However, in absolute terms, the $4 \%$ figure of denture wearers in the JSDF is low. In the Japanese general population, the prevalence of removable denture users in the same age group as our study population is $7.0 \%$ [6] or 9.4\% [22]. These numbers are substantially higher than the result of the current study.

As mentioned above, there is no report on OHRQoL in the other military populations in the literature. When compared with studies on non-military based populations, the frequencies of the impact experienced by our subjects were in general lower than previously reported. For example, the percentage of positive responses to each item ranged from 4.5 to $10.8 \%$ in a Finnish study [23] (age range $30-64$ years), which is higher than our study results. Average OHIP14 summary scores of population-based studies in New Zealand (age 32 years old, male 51.1\%) were 8.0 units [20], 5.1 - 7.7 in Sweden 
(age range 20 - 60 years, male 50\%) [24], 7.1-7.4 in Australia (age <69 years, male 41.4\%) [25], 4.7 to 5.7 in United Kingdom (age <69 years, male 45.7\%) [25], and 2.4 to 4.5 in Finland (age range 30 - 64 years, male 44.3\%) [23], and 11.0 in Malaysia (gender and age data not available) [13]. Once again, although direct comparison is difficult due to the age and gender differences, the OHIP14 summary score in JSDF $(4.6+/-6.7)$ is low in absolute terms compared with other populations. This suggests that JSDF personnel perceive their oral health as only minimally impaired.

The significant association between missing tooth number and OHIP scores was in agreement with previous studies $[2,20,25,26]$, which suggest a patient with more missing teeth is likely to suffer from more OHRQoL impairments. However, and also in agreement with previous studies, the correlation between the key characteristics of physical oral health and how subjects perceive their oral health is not substantial. The prevalence and severity of oral impacts also increased by usage of removable dentures, which is associated with a significant elevation of the OHIP score, as previously reported $[12,23,27]$. It should be noted that the number of missing teeth, which itself has a significant effect on OHRQoL, is larger in those who use removable dentures. Therefore, the presence of removable partial dentures does not necessarily cause impaired OHRQoL. It is just an indicator of impaired OHRQoL. In fact, removable dentures may improve perceived oral health in subjects with missing teeth because of its effect on oral functions such as chewing, speaking, appearance and psychosocial well-being - our study because of its cross-sectional design cannot evaluate the directionality of the denture status-OHRQoL relationship. Tooth loss' impact on OHRQoL can be compensated best with fixed partial dentures or implant dentures. When the number of teeth drops below a certain level and the tooth loss cannot be treated by fixed partial dentures, very likely the removable dentures, even if done to the highest standard in the profession and even if the dentures' quality impact on the OHRQoL [10] is maximized, cannot completely recover lost OHRQoL due to tooth loss. There is a significant cut off point of OHRQoL when a patient moves from the situation where he or she has intact dentition or missing teeth are replaced by fixed partial dentures to the situation where subjects use removable dentures [27]. The clinical implication for military personnel as well as nonmilitary subjects - is that tooth loss should be prevented as much as possible but when it happens, a major deterioration of oral health can be avoided when the magnitude of the tooth loss can still be compensated with fixed prosthodontics and extensive tooth loss, and the use of removable partial denture can be avoided.

\section{Conclusion}

The number of missing teeth and denture status was associated with perceived oral health in the Japanese selfdefense forces. Compared to Japanese civilian populations, personnel of the JSDF demonstrated good oral health.

\section{List of abbreviations}

(OHRQOL): oral health related quality of life; (FPD): fixed partial denture; (RPD): removable partial denture; (OHIP): oral health impact profile; (OHIP-J): OHIP Japanese version; (JSDF): Japan self-defense forces; (CI): confidence interval; (SD): standard deviation

\section{Acknowledgements}

This research was performed at the Department of Prosthodontics, Dental Hospital, Showa University supported by the High-Tech Research Center Project for Private Universities from the Ministry of Education, Culture, Sports, Science, and Technology, Japan, 2005-2009.

\section{Author details}

${ }^{1}$ Department of Prosthodontics, Showa University, Japan. ${ }^{2}$ Department of Diagnostic and Biological Sciences, University of Minnesota, USA.

\section{Authors' contributions}

YK, MTJ, KB, HT and CF conceptualized the rationale and designed the study. YK and CF contributed to the collection of data. MTJ contributed to statistical analysis and interpretation of the data. KB and YS conducted the literature review. All authors read and approved this study.

\section{Competing interests}

The authors declare that they have no competing interests.

Received: 4 October 2010 Accepted: 19 April 2011

Published: 19 April 2011

\section{References}

1. Skec V, Macan JS, Susac M, Jokic D, Brajdic D, Macan D: Influence of oral hygiene on oral health of recruits and professionals in the Croatian Army. Mil Med 2006, 171(10):1006-1009.

2. Pallegedara C, Ekanayake L: Effect of tooth loss and denture status on oral health-related quality of life of older individuals from Sri Lanka. Community Dent Health 2008, 25(4):196-200.

3. Wong MC, McMillan AS: Tooth loss, denture wearing and oral healthrelated quality of life in elderly Chinese people. Community Dent Health 2005, 22(3):156-161.

4. Jones JA, Orner MB, Spiro A, Kressin NR: Tooth loss and dentures: patients' perspectives. Int Dent J 2003, 53(5 Suppl):327-334.

5. McGrath C, Bedi R: Can dentures improve the quality of life of those who have experienced considerable tooth loss? J Dent 2001, 29(4):243-246.

6. Ide R, Yamamoto R, Mizoue T: The Japanese version of the Oral Health Impact Profile (OHIP)-validation among young and middle-aged adults. Community Dent Health 2006, 23(3):158-163.

7. Slade GD, Spencer AJ: Development and evaluation of the Oral Health Impact Profile. Community Dent Health 1994, 11(1):3-11.

8. Baba K, Igarashi Y, Nishiyama A, John MT, Akagawa Y, Ikebe K, Ishigami T, Kobayashi H, Yamashita S: Patterns of missing occlusal units and oral health-related quality of life in SDA patients. J Oral Rehabil 2008, 35(8):621-628

9. Baba K, John MT, Inukai M, Aridome K, Igarahsi Y: Validating an alternate version of the chewing function questionnaire in partially dentate patients. BMC Oral Health 2009, 9:9.

10. Inukai $M$, Baba $K$, John MT, Igarashi $Y$ : Does removable partial denture quality affect individuals' oral health? J Dent Res 2008, 87(8):736-739.

11. Rener-Sitar K, Petricevic N, Celebic A, Marion L: Psychometric properties of Croatian and Slovenian short form of oral health impact profile questionnaires. Croat Med J 2008, 49(4):536-544.

12. John MT, Miglioretti DL, LeResche L, Koepsell TD, Hujoel P, Micheelis W: German short forms of the Oral Health Impact Profile. Community Dent Oral Epidemiol 2006, 34(4):277-288. 
13. Saub R, Locker D, Allison P: Derivation and validation of the short version of the Malaysian Oral Health Impact Profile. Community Dent Oral Epidemiol 2005, 33(5):378-383.

14. Slade GD: Derivation and validation of a short-form oral health impact profile. Community Dent Oral Epidemiol 1997, 25(4):284-290.

15. Yamazaki M, Inukai M, Baba K, John MT: Japanese version of the Oral Health Impact Profile (OHIP-J). J Oral Rehabil 2007, 34(3):159-168.

16. Baba K, Inukai M, John MT: Feasibility of oral health-related quality of life assessment in prosthodontic patients using abbreviated Oral Health Impact Profile questionnaires. J Oral Rehabil 2008, 35(3):224-228.

17. Zadik Y, Zusman SP, Galor S, Dinte AF: Dental attendance and selfassessment of dental status by Israeli military personnel according to gender, education, and smoking status, 1998-2006. Mil Med 2009, 174(2):197-200.

18. Marker OT, Vigild M, Praetorius F: Oral health problems and treatment needs in Danish military personnel recruited for United Nations service. Mil Med 1997, 162(6):416-421.

19. Cohen J: Statistical Power Analysis for the Behavioral Sciences 2 edition. Hillsdale: Lawrence Erlbaum Associates; 1988.

20. Lawrence HP, Thomson WM, Broadbent JM, Poulton R: Oral health-related quality of life in a birth cohort of 32-year olds. Community Dent Oral Epidemiol 2008, 36(4):305-316.

21. Acharya S: Oral health-related quality of life and its associated factors in an Indian adult population. Oral Health Prev Dent 2008, 6(3):175-184.

22. Ministry of Health, Labour and Welfare Japan: Statistical Tables of The Survey of Dental Disease. 2005 [http://www.mhlw.go.jp/topics/2007/01/ tp0129-1.html].

23. Lahti S, Suominen-Taipale L, Hausen $\mathrm{H}$ : Oral health impacts among adults in Finland: competing effects of age, number of teeth, and removable dentures. Eur J Oral Sci 2008, 116(3):260-266.

24. Einarson $S$, Gerdin EW, Hugoson A: Oral health impact on quality of life in an adult Swedish population. Acta Odontol Scand 2009, 67(2):85-93.

25. Steele JG, Sanders AE, Slade GD, Allen PF, Lahti S, Nuttall N, Spencer AJ: How do age and tooth loss affect oral health impacts and quality of life? A study comparing two national samples. Community Dent Oral Epidemiol 2004, 32(2):107-114.

26. Sanders AE, Slade GD, Lim S, Reisine ST: Impact of oral disease on quality of life in the US and Australian populations. Community Dent Oral Epidemiol 2009, 37(2):171-181.

27. John MT, Koepsell TD, Hujoel P, Miglioretti DL, LeResche L, Micheelis W: Demographic factors, denture status and oral health-related quality of life. Community Dent Oral Epidemiol 2004, 32(2):125-132.

\section{Pre-publication history}

The pre-publication history for this paper can be accessed here: http://www.biomedcentral.com/1472-6831/11/14/prepub

doi:10.1186/1472-6831-11-14

Cite this article as: Kudo et al:: Oral health in the Japan self-defense forces - a representative survey. BMC Oral Health 2011 11:14.

\section{Submit your next manuscript to BioMed Central and take full advantage of:}

- Convenient online submission

- Thorough peer review

- No space constraints or color figure charges

- Immediate publication on acceptance

- Inclusion in PubMed, CAS, Scopus and Google Scholar

- Research which is freely available for redistribution

Submit your manuscript at www.biomedcentral.com/submit
Biomed Central 